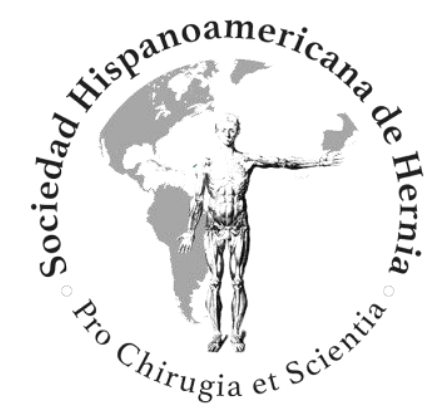

Técnica modificada rectus turnover flap para hernia ventral compleja en paciente con pérdida de domicilio y obesidad mórbida

\title{
Modified rectus turnover flap technique for complex ventral hernia in a patient with loss of domain and morbid obesity
}




\section{424}

\section{Técnica modificada rectus turnover flap para hernia ventral compleja en paciente con pérdida de domicilio y obesidad mórbida}

Modified rectus turnover flap technique for complex ventral hernia in a patient with loss of domain and morbid obesity

Andrés Felipe Escudero Sepúlveda ${ }^{1}$, Romina Pinasco ${ }^{1}$, Miguel Iván Rodríguez², Julián Camilo Cala Durán ${ }^{3}$, Fabián Leonardo Escudero Sepúlveda ${ }^{4}$, Gonzalo Andrés Domínguez-Alvarado ${ }^{5}$, Leydis Vanesa Pérez Urrea ${ }^{2}$

${ }^{1}$ Servicio de Cirugía General, Laparoscópica y Miniinvasiva. Hospital de Trauma y Emergencias Dr. Federico Abete. Gran Buenos Aires (Argentina). ${ }^{2}$ Fundación Oftalmológica de Santander Clínica Carlos Ardila Lülle. Floridablanca (Colombia). ${ }^{3}$ Servicio de Vigilancia Epidemiológica. Hospital Universitario de Santander. Bucaramanga, Santander (Colombia). ${ }^{4}$ Medicina Familiar. IPS SURA. Universidad Autónoma de Bucaramanga. Rionegro (Colombia). ${ }^{5}$ Servicio Social Obligatorio. Clínica Foscal Internacional. Semillero de Innovación e Investigación en Cirugía (SIIC). Floridablanca, Santander (Colombia)

Recibido: 06-01-2021

Aceptado: 21-04-2021

Autor para correspondencia: Andrés Felipe Escudero Sepúlveda. Servicio de Cirugía General, Laparoscópica y Miniinvasiva. Hospital de Trauma y Emergencias Dr. Federico Abete. 123, Miraflores. Pablo Nogués, Provincia de Buenos Aires (Argentina)

Correo electrónico: dr.andresescudero@gmail.com

DOI: $10.20960 /$ rhh.00424 
Conflicto de interés: los autores declaran no tener conflicto de intereses. Protección de personas y animales: los autores declaran que para este reporte no se han realizado experimentos en seres humanos ni en animales.

Confidencialidad de los datos: los autores declaran que han seguido los protocolos de su centro de trabajo sobre la publicación de datos de pacientes.

Derecho a la privacidad y consentimiento informado: los autores declaran ha obtenido el consentimiento informado del paciente y/o sujetos referidos en el artículo. Este documento obra en poder del autor de correspondencia.

\section{RESUMEN}

Introducción: La hernia incisional es una frecuente y desafiante patología para el cirujano general, con una incidencia entre el 9 al $69 \%$, el conocimiento de los elementos y habilidades técnicas para su reparación se convierten en indispensables en la cirugía de pared abdominal. Se presentan dos variaciones de la técnica rectus turnover flap para la reparación de hernias de línea media, donde se usa malla en posición sublay asociada a una separación de componentes posterior.

Caso clínico: Paciente de 52 años, con antecedente de laparotomía por posterior complicación de 2 años de evolución con hernia ventral compleja, pérdida de domicilio y obesidad mórbida (IMC $43.1 \mathrm{~kg} / \mathrm{m}^{2}$ ) al momento de la reparación. Requirió de la realización de neumoperitoneo preoperatorio con 9 litros de aire ambiente; presentó complicación leve (necrosis parcial de la herida) que se resuelve con el manejo de colagenasa tópica y control posoperatorio a los 12 meses sin evidencia, al examen físico, de defecto de pared abdominal.

Conclusión: En casos donde se requiere de la movilización de tejido para poder restaurar la línea media las dos modificaciones de la técnica rectus 
turnover flap, pueden convertirse en una herramienta más para los cirujanos de pared abdominal.

Palabras clave: Hernia ventral, obesidad, pérdida de domicilio, técnica rectus turnover flap, neumoperitoneo preoperatorio, separación de componentes posterior.

\section{ABSTRACT}

Introduction: Incisional hernia is a frequent and challenging pathology for general surgeons, with an incidence between $9 \%$ and $69 \%$; the knowledge of elements and technical skills for its repair become an indispensable tool in abdominal wall surgery.

Two variations of the rectus turnover flap technique are presented for the repair of midline hernias, where mesh is used in sublay position associated with a posterior component separation.

Case report: 52-year-old patient with a history of laparotomy due to a posterior complication of 2 years of evolution with complex ventral hernia, loss of residence and morbid obesity (BMI $43.1 \mathrm{~kg} / \mathrm{m}^{2}$ ) at the time of repair. It required a preoperative pneumoperitoneum with 9 liters of ambient air. He presented a mild complication (partial necrosis of the wound) that resolved with management of topical collagenase and postoperative control at 12 months without evidence of an abdominal wall defect upon physical examination.

Conclusion: In cases in which tissue mobilization is required to restore the midline, the modifications to the rectus turnover flap technique can be yet another tool for the abdominal wall surgeon.

Keywords: Ventral hernia, obesity, loss of domian hernia, rectus turnover flap, preoperative pneumoperitoneum, posterior component separation.

\section{INTRODUCCIÓN}

La hernia incisional es una patología frecuente posterior a la realización de una laparotomía con una incidencia entre el 9 al $69 \%{ }^{1,2}$, la cual se ve 
afectada por el tipo de cirugía y las características de los pacientes ${ }^{1}$. Se propuso para disminuir su incidencia pautas para el cierre de una laparotomía definidas por la Sociedad Europea de la Hernia (EHS) ${ }^{3}$. El sobrepeso y la obesidad son factores de riesgo que aumentan la morbimortalidad de los pacientes y se asocian a trastornos musculoesqueléticos ${ }^{4}$. La reducción entre el 2-5\% y > $5 \%$ del peso en los pacientes se asocia a mejoría de sus patologías de base ${ }^{4}$. Un aumento en el índice de masa corporal (IMC) da como resultado mayor probabilidad para la complicación de la herida, de ahí la importancia de la reducción del peso preoperatoriamente ${ }^{5}$.

Lejos estamos de tener una técnica ideal, razón por la cual el cirujano de pared abdominal debe conocer y estar familiarizado con los recursos disponibles, las técnicas quirúrgicas, materiales protésicos, las técnicas de explicación tisular ${ }^{2,3,6,7}$ y el respeto de los principios para la reparación de la pared abdominal ${ }^{8}$.

\section{CASO CLÍNICO}

Paciente de 51 años, de profesión vendedor de productos veterinarios, hipertenso controlado, obeso mórbido con peso de $138 \mathrm{~kg}$ e IMC de 45.06 $\mathrm{kg} / \mathrm{m}^{2}$ y con antecedente, hace 2 años, de laparotomía exploradora. Se presenta a consulta con eventración supra infraumbilical de aparición a los 2 meses de posoperatorio (POP), asociada a síntomas de constipación, dolor lumbar y limitación para realizar actividades laborales de levantamiento y transporte de bolsas de alimentos de $20 \mathrm{~kg}$ de peso.

Al examen físico se observa abdomen con cicatriz supra infraumbilical, defecto de pared de $12 \mathrm{~cm}$ en eje longitudinal y $23 \mathrm{~cm}$ en eje transverso y contenido abdominal con pérdida de domicilio.

Se solicita tomografía computarizada de abdomen y pelvis con doble contraste con cálculo de índice de Tanaka. Los resultados reportan defecto de pared abdominal a nivel medial con anillo de $13.5 \mathrm{~cm} \times 24.1 \mathrm{~cm}$, con contenido de asas intestinales y colon, sin signos de oclusión, con índice de Tanaka de $37.4 \%$ y con espirometría sin alteraciones. Se solicitan exámenes prequirúrgicos, remisión a nutrición para descenso de peso y 
programar internación para realización intrahospitalaria de neumoperitoneo. Se clasifica como una hernia incisional M2-4 W3 de la EHS.

El paciente ingresa para internación dos meses después con peso de 132 $\mathrm{kg}$ y IMC $43.1 \mathrm{~kg} / \mathrm{m}^{2}$, se inicia neumoperitoneo con colocación de catéter de vía central en punto de McBurney izquierdo, con insuflaciones diarias por 15 días con volúmenes entre 500 y 1000 cc diarios de aire ambiente, para un total 9 litros de aire ambiente.

Una vez el paciente se encontraba en condiciones, se decide llevar a quirófano en plan de realización de hernioplastia con malla intraperitoneal, asociada a variación de la técnica de rectus turnover flap (fig. 1). Se realiza marcación del abdomen, anestesia general, cefalotina 2 g EV, previo a incisión por preferencia personal para facilitar la disección del saco herniario; se implementó la aplicación de aire ambiente entre la piel y el saco, utilizando una aguja de $14 \mathrm{~F}$, llave de tres vías y jeringa de 60 cc; después se realizó incisión en losange sobre saco herniario, disección del saco sin apertura del mismo, con técnica de transiluminación. Se identifica área de acceso seguro a la pared (fig. 2) apertura medial del saco y liberación del neumoperitoneo, enterólisis y adhesiólisis, se realiza incisión sobre la lámina anterior del recto a nivel lateral, posterior separación y rotación del recto bilateralmente (fig. 3). Se evidencia que aun realizando esta maniobra no es posible restituir la línea media, por lo que se opta por la realización de la separación de componentes posterior tipo TAR (fig. 4); se realiza separación del oblicuo menor y el músculo transverso, y resección de las fibras musculares del músculo transverso abdominal (fig. 5); se verifica con maniobra de cruzamiento de Allis que se logra llevar los bordes a línea media sin tensión (fig. 6) y se coloca malla de contacto visceral con doble reparo de puntos de proliglactina 2/0, similar a la técnica de fijación en doble corona. Posteriormente con sutura continua se afronta en línea media las láminas anteriores de los rectos con poliglantina 0 y puntos separados para afrontar músculos rectos con poliglactina 0 (fig. 7). Se anudan las dos líneas reparadas en puntos cardinales de la malla. Colocación de dos drenajes a tejido celular 
subcutáneo. Se realiza resección del excedente de piel. Cierre con puntos de poliglactina $2 / 0$ a tejido celular subcutáneo y piel con prolene $2 / 0$ puntos separados.

El paciente pasa a planta para control clínico POP y manejo de dolor. Egreso con drenajes al segundo día con tolerancia de dieta general. En control por consultorio, al quinto día, se evidencia necrosis parcial de la herida a nivel caudal y se indica desbridamiento con colagenasa tópica y control a los 10 días donde se evidencia mejoría de la herida y del tejido de granulación. El cierre definitivo de la herida se presenta a los 30 días de POP (fig. 8).

El paciente acude a control al 6. mes de POP con disminución de peso de $16 \mathrm{~kg}$ y con la herida cicatrizada; se realiza una exploración física y de maniobras de Valsalva y no se evidencian defectos en la pared abdominal (fig. 9). Refiere poder realizar actividad laboral sin problemas y actividades de peso con faja elástica; también presenta mejoría de la constipación y dolor lumbar.

\section{DISCUSIÓN}

La hernia se define como un defecto de la aponeurosis, que de asociarse al antecedente de una cirugía se denomina como hernia incisional, hernia secundaria o eventración ${ }^{9}$.

La frecuencia de hernia incisional en los pacientes obesos es del $2 \%$ al $11 \%$ con altas tasas de recurrencia del $20 \%$ al $30 \%{ }^{10}$. La probabilidad de presentar una complicación en un paciente con IMC > $35 \mathrm{~kg} / \mathrm{m}^{2}$ es 1.5 veces mayor que en un paciente con normopeso. Se encontró una asociación del IMC de $30 \mathrm{~kg} / \mathrm{m}^{2}$ con infección de herida quirúrgica (SSI) superficial (OR 1.82), SSI profunda (OR 2.87) y dehiscencia de la herida $(\mathrm{OR} 4.5)^{11}$. La probabilidad de presentar hernia incisional por infección del sitio quirúrgico en un paciente obeso alcanza el $40 \%{ }^{12}$. El paciente presentó dehiscencia y necrosis parcial de la herida que mejoró con el manejo con desbridamiento con colagenasa tópica. 
Actualmente se disponen de técnicas quirúrgicas, mallas y elementos diagnósticos; el uso de la malla puede llevar a disminuir a un $5 \%$ la frecuencia de esta complicación ${ }^{12}$.

La pérdida de domicilio hace referencia a la permanencia del contenido abdominal en el saco herniario, comportándose como una segunda cavidad abdominal ${ }^{13}$. De manera objetiva se puede hallar el índice de Tanaka, que es la relación volumétrica entre la cavidad abdominal y el volumen de defecto herniario, cuando esta relación es $>15-20 \%$ se habla cuantitativamente de pérdida de domicilio. Se considera que con un índice de Tanaka $\geq 25 \%$ el paciente es candidato o estaría indicado para la realización de neumoperitoneo preoperatorio ${ }^{13-15}$.

La preparación de la cavidad abdominal propuesta por Goñi Moreno permite la adaptación fisiológica del paciente y el reintegro de las vísceras a la cavidad abdominal. De este modo disminuye el riesgo de presentar un síndrome compartimental, como producto del reintegro del contenido herniado a la cavidad abdominal, con aumento súbito de la presión intraabdominal y posterior compromiso cardiopulmonar ${ }^{16}$. El $25 \%$ de los pacientes obesos con hernias abdominales con pérdida de domicilio pueden experimentar alguna complicación posoperatoria grave, entre estas la insuficiencia respiratoria aguda posoperatoria, razón por la cual la adaptación con PPP es fundamental en la planificación quirúrgica ${ }^{10}$.

Para la clasificación de la hernia incisional se usa como referencia la sugerida por la $\mathrm{EHS}^{17}$ al igual que los principios de cierre de la pared abdominal ${ }^{3}$.

DeFranzo y cols. en 1996 presentaron la técnica de rectus turnover flap para la reconstrucción de los defectos de línea media de la pared abdominal, que consiste en la liberación de la lámina anterior de los músculos rectos y la rotación hacia medial de los mismos, con la utilización de una malla supraaponeurótica (onlay) ${ }^{18}$. Se presentan dos modificaciones a la técnica, ambas con el uso de malla sintética no absorbible con barrera de celulosa regenerada oxidada ${ }^{7}$, en posición intraperitoneal (sublay); en la primera la fijación se realiza con sutura absorbible a nivel de la desinserción de la lámina anterior del músculo 
recto (fig. 1). En la segunda modificación se asocia la técnica a una separación de componentes posterior (posterior component separation) tipo transversus abdominis muscle release $(\mathrm{TAR})^{19}$. Para su fijación se utilizó una doble línea de suturas, agregando una sutura más al borde del músculo trasverso y tomando el borde de las aponeurosis de los músculos oblicuos; así se sitúan en una nueva posición anatómica y se evita la retracción de los mismos a lateral (fig. 4).

Esta técnica respeta los principios expuestos por Daes y Telem para la reparación de la hernia ventral ${ }^{8}$ :

1. Cierre primario de defectos faciales bajo tensión fisiológica cuando sea posible.

2. Amplio refuerzo protésico del saco visceral.

3. Fijación limitada de material protésico.

4. Es preferible la colocación de mallas por subcapa, aunque es aceptable una colocación alternativa.

5. Abordaje mínimamente invasivo (IM) cuando sea posible.

6. Prehabilitación del paciente.

7. Protocolo de recuperación mejorada después de la cirugía (ERAS).

Existen otros elementos para la expansión tisular, además del neumoperitoneo preoperatorio (PPP), como la toxina botulínica y los implantes expansores de tejidos, pero no se disponen en el lugar de actividad quirúrgica. Cabe aclarar que en el $51.7 \%$ de los pacientes se asoció la toxina botulínica a separación de componentes y solo se logró el cierre primario en el $34.5 \%$ de los pacientes en comparación al $84 \%$ de los pacientes con PPP2.

\section{CONCLUSIÓN}

Se presenta una alternativa quirúrgica para la resolución de la hernia incisional compleja de gran tamaño con pérdida de domicilio, que puede ser utilizada en pacientes complejos por sus patologías de base.

Las dos modificaciones de la técnica rectus turnover flap, pueden convertirse en una herramienta más para los cirujanos de pared 
abdominal, en casos donde se requiere de la movilización de tejido para poder restaurar la línea media.

Es importante la preparación prequirúrgica del paciente para reducir el riesgo de complicaciones posoperatorias. Familiarizarse con los recursos disponibles en la actualidad es un deber del cirujano que maneja patología compleja de la pared abdominal.

\section{BIBLIOGRAFÍA}

1. Henriksen NA, Deerenberg EB, Venclauskas L, Fortelny $R H$, Miserez $M$, Muysoms FE. Meta-analysis on Materials and Techniques for Laparotomy Closure: The MATCH Review. World J Surg. 2018;42(6):1666-78. DOI: 10.1007/s00268-017-4393-9

2. Alam NN, Narang SK, Pathak S, Daniels IR, Smart NJ. Methods of abdominal wall expansion for repair of incisional herniae: a systematic review. Hernia. 2016;20(2):191-9. DOI: 10.1007/s10029-016-1463-0

3. Burger JWA, Luijendijk RW, Hop WCJ, Halm JA, Verdaasdonk EGG, Jeekel J, et al. Long-term follow-up of a randomized controlled trial of suture versus mesh repair of incisional hernia. Ann Surg. 2004;240(4):578-85. DOI: 10.1097/01.sla.0000141193.08524.e7

4. Caiña OD, Escudero Sepúlveda AF, Aguilar J, Martin SJ, Maestre JA, Camilo J, et al. Caracterización y Evolución Clínica a 12 meses en pacientes con Obesidad y Diabetes tipo 2 sometidos a Cirugía Bariátrica mediante técnica de Bypass Gástrico de una Anastomosis. Bariátrica \& Metabólica Ibero-Americana. 2019;9.2.5:2566-75.

5. Tastaldi L, Krpata DM, Prabhu AS, Petro CC, Rosenblatt S, Haskins IN, et al. The effect of increasing body mass index on wound complications in open ventral hernia repair with mesh. Am J Surg. 2019;218(3):560-6. DOI: 10.1016/j.amjsurg.2019.01.022

6. López-casillas N, Félix-álvarez CA, Armando H, Muñoz C, Carlos J, González M. Manejo de hernias incisionales complejas: experiencia en un hospital de segundo nivel Management of complex incisional hernias: experience in a second level hospital of attention. Rev Hispanoam Hernia. 2020;8(2):56- 
64. DOI: 10.20960/rhh.00229

7. López-Cano M, Barreiro Morandeira F. Prótesis en el tratamiento de las eventraciones. Cir Esp. 2010;88(3):152-7. DOI: 10.1016/j.ciresp.2009.12.015

8. Daes J, Telem D. The Principled Approach to Ventral Hernia Repair. Rev Colomb Cir. 2019;34:25-8. DOI: 10.30944/20117582.94

9. Domínguez Caro R, Medrano Caviedes R, Rofin Serra S, Caballero Mestres F, Trias Folch M. Cirugía urgente de la pared abdominal. Rev Hispanoam Hernia. 2017;3(5):91-4. DOI: 10.20960/rhh.97

10. Valezi AC, de Melo BGF, Marson AC, Liberatti M, Lopes AG. Preoperative progressive pneumoperitoneum in obese patients with loss of domain hernias. Surg Obes Relat Dis. 2018;14(2):138-42. DOI: 10.1016/j.soard.2017.10.009

11. Kaoutzanis C, Leichtle SW, Mouawad NJ, Welch KB, Lampman RM, Wahl $W L$, et al. Risk factors for postoperative wound infections and prolonged hospitalization after ventral/incisional hernia repair. Hernia. 2015;19(1):113-23. DOI: 10.1007/s10029-013-1155-y

12. Derlin Juárez M. Manejo multimodal de la eventración gigante. Rev Hispanoam Hernia. 2018;6(1):3-10. DOI: 10.20960/rhh.57

13. Kingsnorth AN, Sivarajasingham N, Wong S, Butler M. Open mesh repair of incisional hernias with significant loss of domain. Ann R Coll Surg Engl. 2004;86(5):363-6. DOI: 10.1308/147870804236

14. Tanaka EY, Yoo JH, Rodrigues AJ, Utiyama EM, Birolini D, Rasslan S. A computerized tomography scan method for calculating the hernia sac and abdominal cavity volume in complex large incisional hernia with loss of domain. Hernia. 2010;14(1):63-9. DOI: 10.1007/s10029-009-0560-8

15. Parker SG, Halligan S, Blackburn S, Plumb AAO, Archer L, Mallett S, et al. What Exactly is Meant by "Loss of Domain" for Ventral Hernia? Systematic Review of Definitions. World J Surg. 2019;43:396-404. DOI: 10.1007/s00268-018-4783-7

16. López Sanclemente MC, Robres J, López Cano M, Barri J, Lozoya R, López S, et al. Progressive preoperative pneumoperitoneum in patients with giant hernias of the abdominal wall. Cir Esp. 2013;91(7):444-9. DOI: 
10.1016/j.cireng.2012.08.001

17. Marenco de la Cuadra B, Guadalajara Jurado JF, García Moreno J, Moreno Romero R. Actualización Clasificación actual EHS. Cir Andal. 2013;24:2257.

18. DeFranzo AJ, Kingman GJ, Sterchi MJ, Marks MW, Thorne M. Rectus Turnoover Flaps for the Reconstruction of Large Modline abdominal Wall Defects. Ann Plast Surg. 1996;37(1):18-23. DOI: 10.1097/00000637199607000-00003

19. Novitsky YW, Elliott HL, Orenstein SB, Rosen MJ. Transversus abdominis muscle release: A novel approach to posterior component separation during complex abdominal wall reconstruction. Am J Surg. 2012;204(5):709-16. DOI: 10.1016/j.amjsurg.2012.02.008

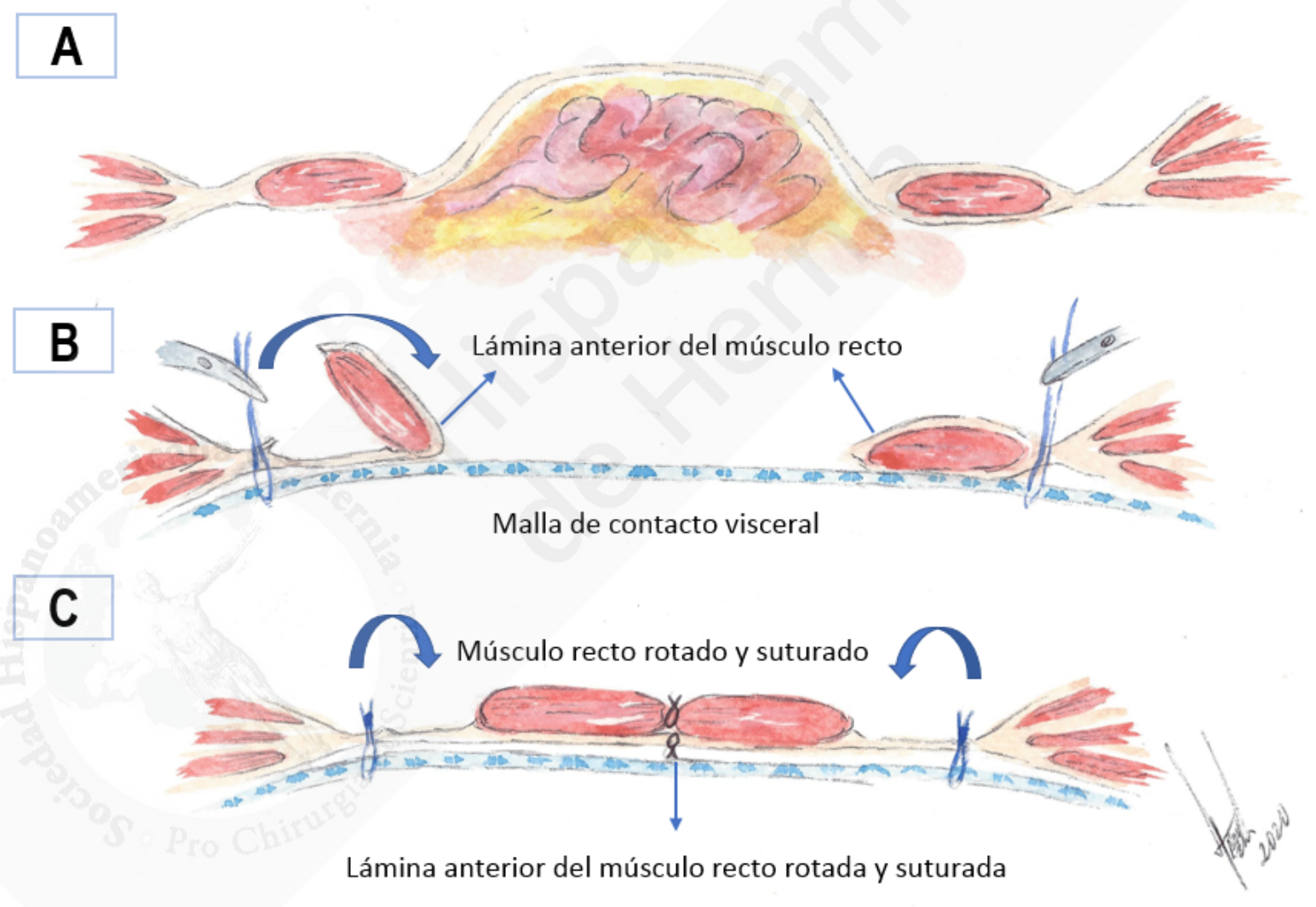

Figura 1. Modificación de la técnica de rectus turnover flap. A. Disposición prequirúrgica de los planos anatómicos. B. Incisión a de la lámina anterior del recto en su borde lateral y posterior rotación hacia medial del musculo 
recto. Puntos cardinales de la malla de contacto visceral reparados. C. Músculos rotados y suturados en línea media, laminas anteriores suturadas en línea media, malla intraperitoneal fijada.

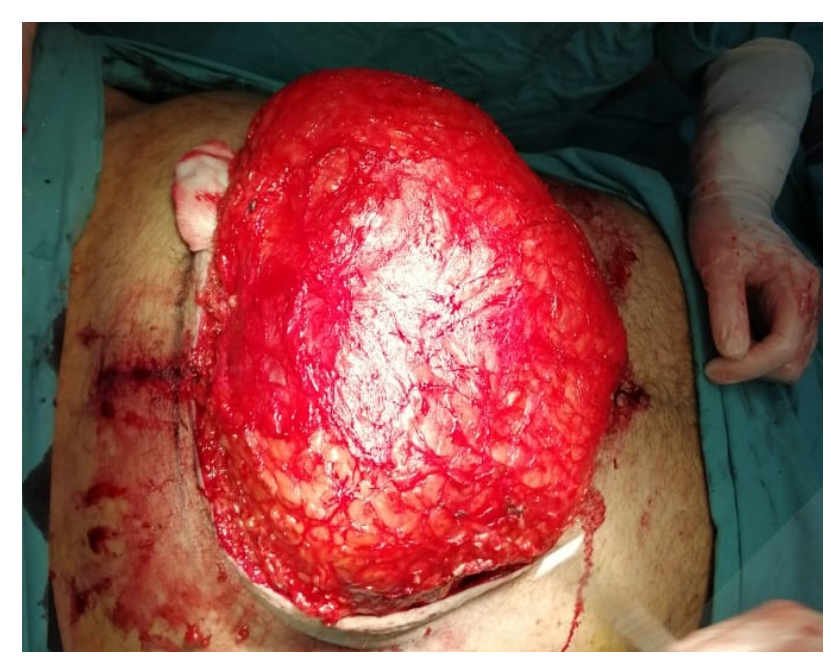

Figura 2. Saco herniario disecado, con neumoperitoneo sin evacuar, se logra evidenciar el importante defecto herniario que padece el paciente.

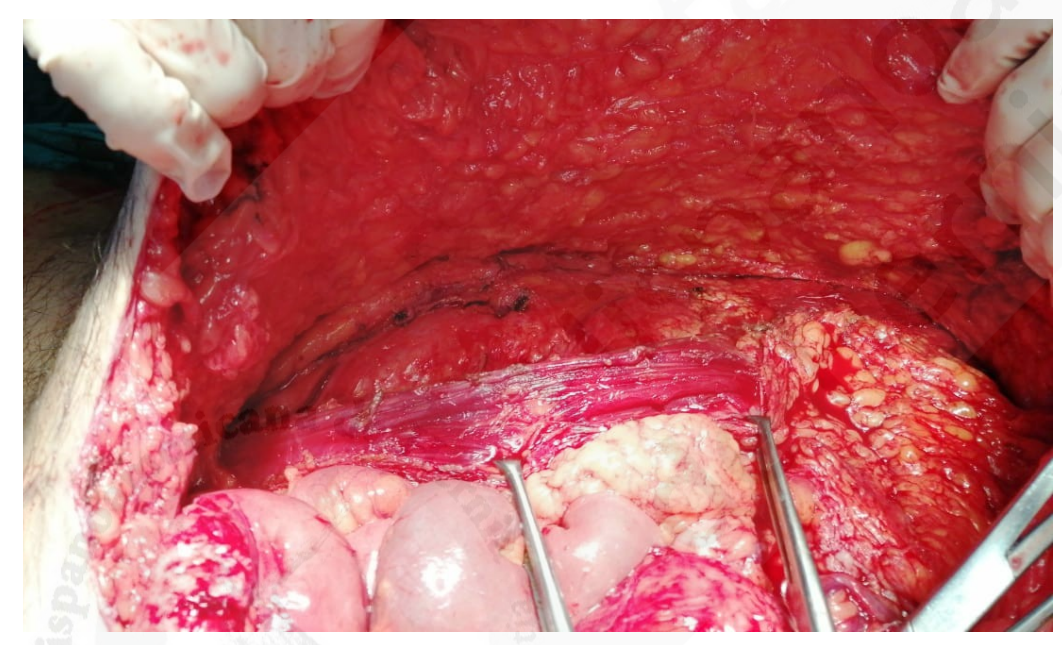

Figura 3. Se evidencia borde de la lámina anterior del recto, seccionado a nivel lateral y rotación del músculo recto hacia medial. 


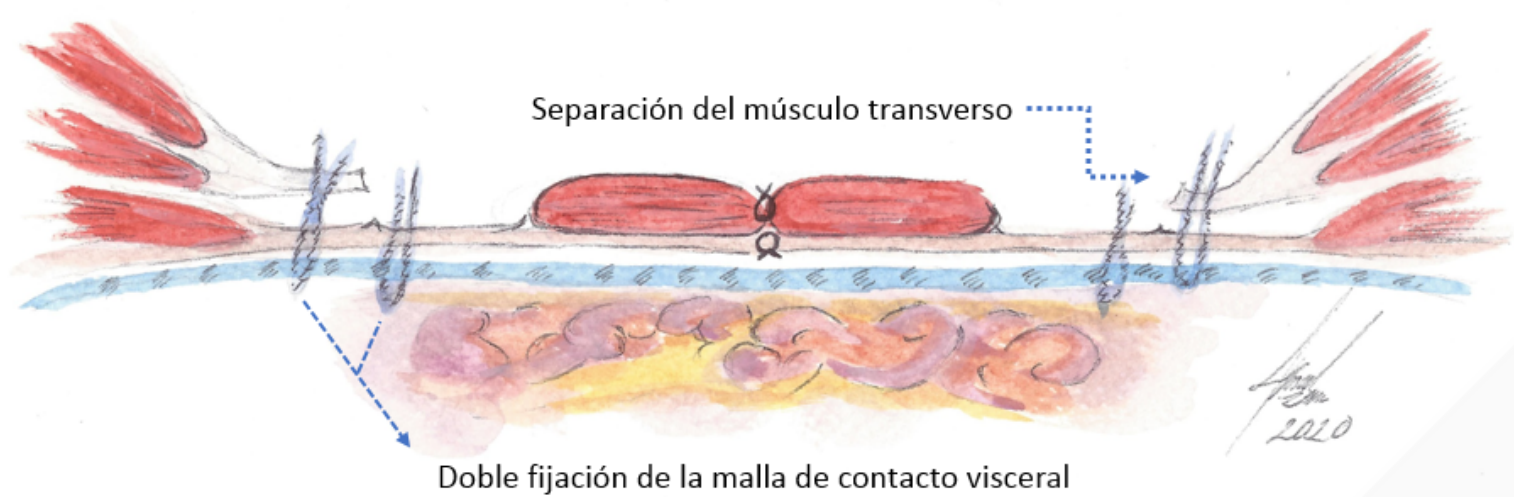

Figura 4. Modificación de la técnica de rectus turnover flap asociado a separación de componentes posterior. Se conserva separación y liberación de las fibras del músculo transverso abdominal y la doble línea de fijación de la malla de contacto visceral.

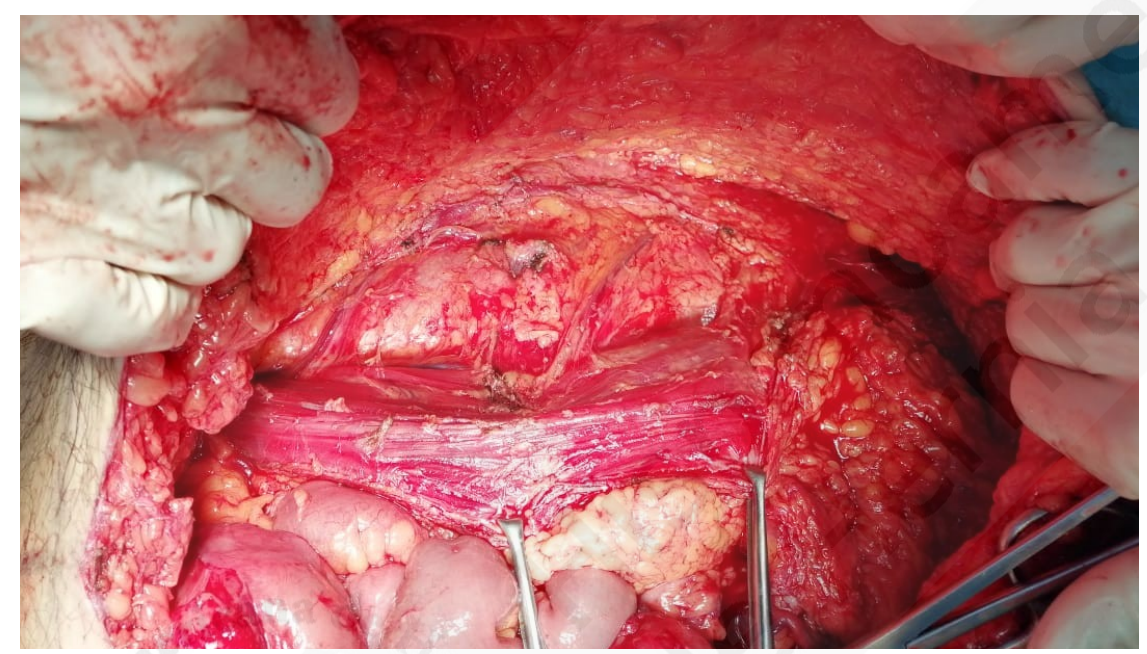

Figura 5. Se observa músculo recto rotado y se realiza la separación de componentes posterior-TAR. 


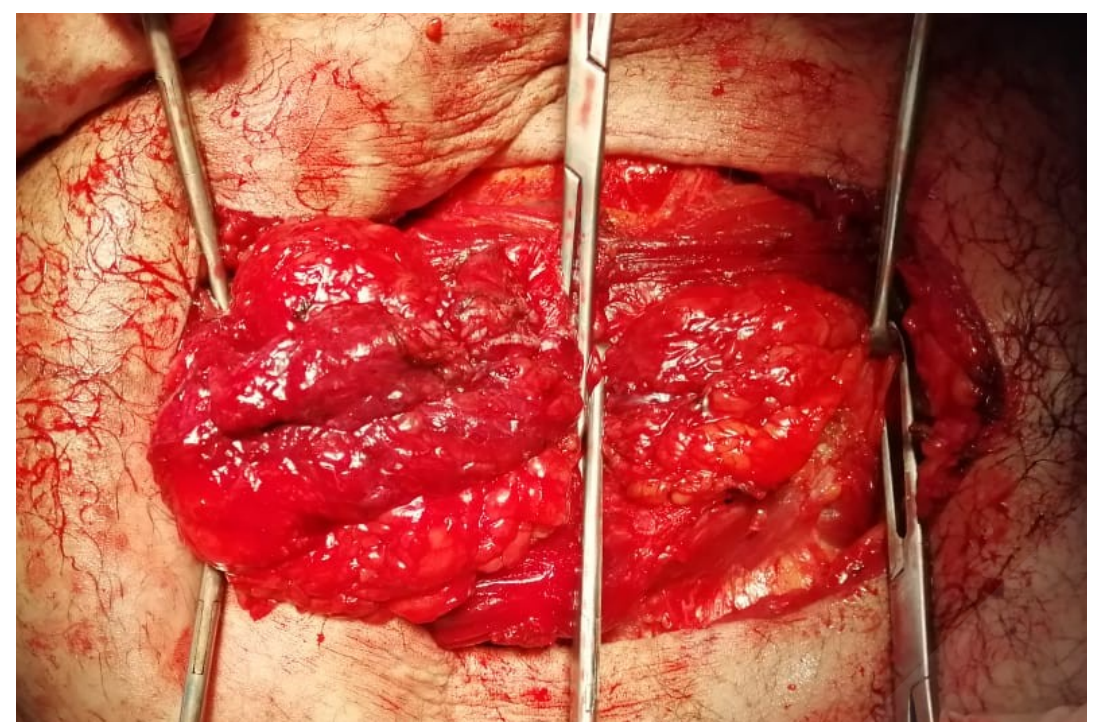

Figura 6. Se comprueba, con la utilización de pinzas Allis cruzadas, la posibilidad de restablecer la línea media.

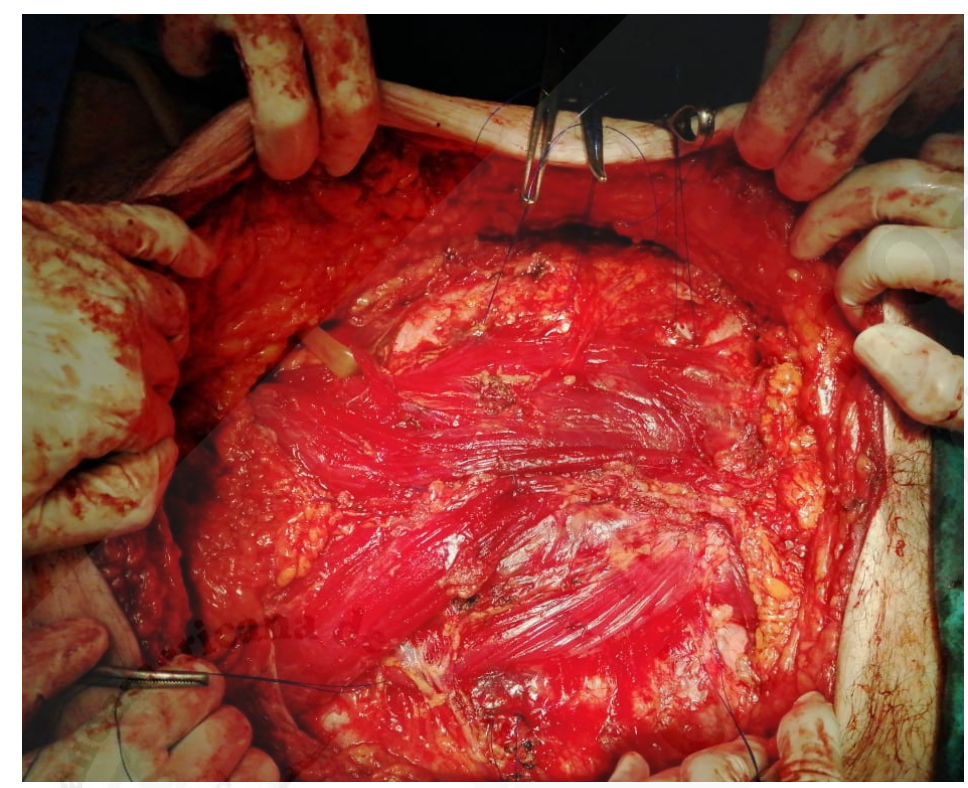

Figura 7. Se observa músculo recto rotado y suturado en línea media. Lámina posterior del recto y los reparos de las dos líneas de sutura de la malla de contacto visceral. 

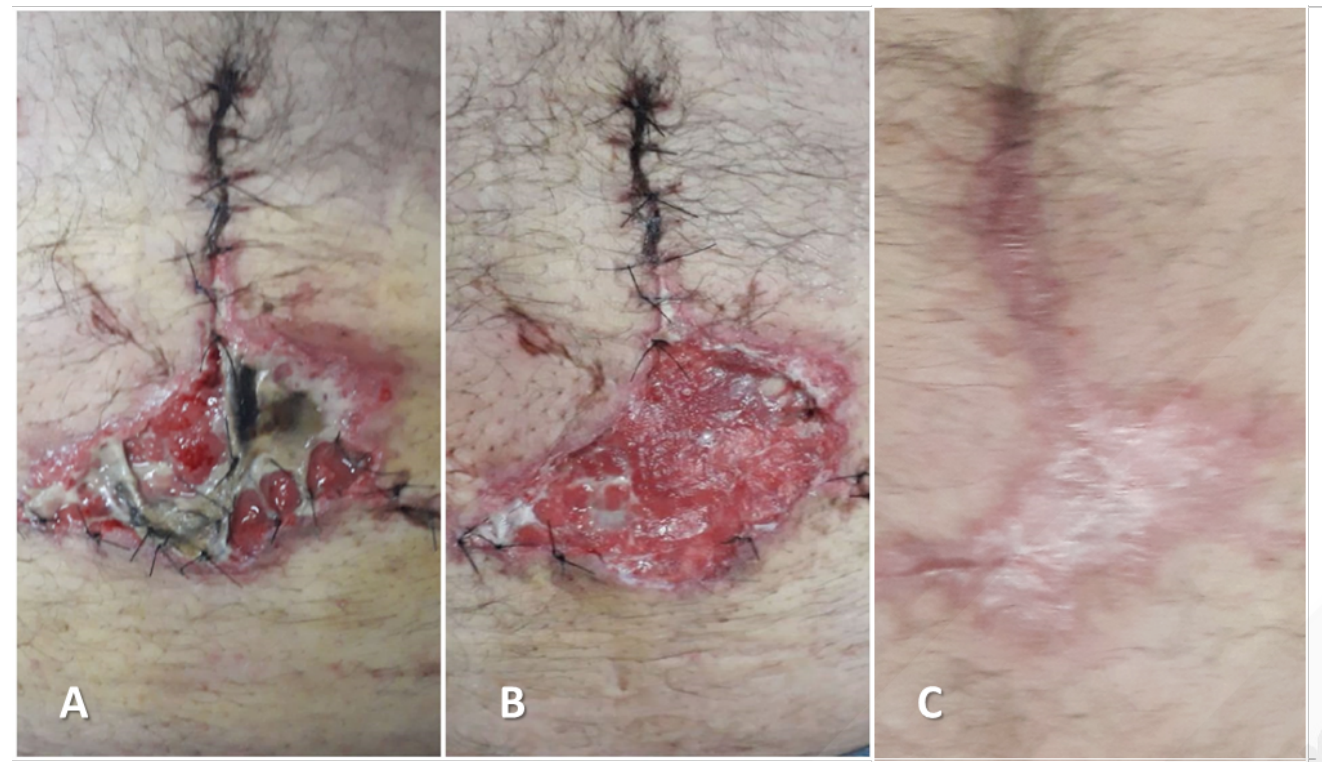

Figura 8. A. 5. día POP. Necrosis parcial de la piel de la herida a nivel caudal. B. 10. o día POP. Se observa tejido de granulación posterior al desbridamiento con colagenasa. C. 30 días POP. Herida cicatrizada.

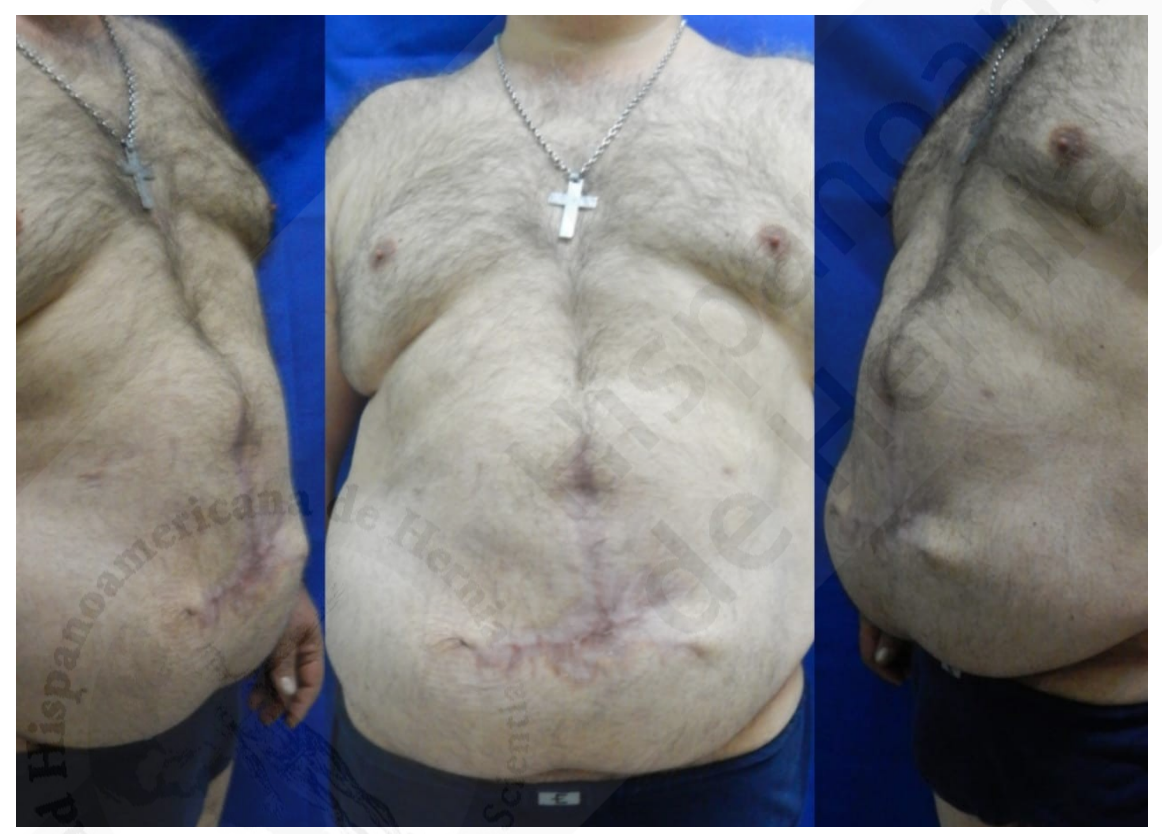

Figura 9. Vista lateral y frontal del paciente a 6 meses de posoperatorio, realizando maniobra de Valsalva, sin evidencia de defecto en la pared abdominal. 